\title{
UNIFORM ALGEBRAS GENERATED BY HOLOMORPHIC AND PLURIHARMONIC FUNCTIONS
}

\author{
ALEXANDER J. IZZO
}

\begin{abstract}
It is shown that if $f_{1}, \ldots, f_{n}$ are pluriharmonic on $B_{n}$ (the open unit ball in $\left.\mathbb{C}^{n}\right)$ and $C^{1}$ on $\bar{B}_{n}$, and the $n \times n$ matrix $\left(\partial f_{j} / \partial \bar{z}_{k}\right)$ is invertible at every point of $B_{n}$, then the norm-closed algebra generated by the ball algebra $A\left(\bar{B}_{n}\right)$ and $f_{1}, \ldots, f_{n}$ is equal to $C\left(\bar{B}_{n}\right)$. Extensions of this result to more general strictly pseudoconvex domains are also presented.
\end{abstract}

\section{INTRODUCTION}

For $K$ a compact set in $\mathbb{C}^{n}$, let $A(K)$ denote the subalgebra of $C(K)$ consisting of the continuous functions on $K$ that are holomorphic on the interior of $K$. Let $D$ denote the open unit disc in the plane. It is known that if $f$ is in $C(\bar{D})$ and $f$ is harmonic but not holomorphic on $D$, then the norm-closed subalgebra of $C(\bar{D})$ generated by the disc algebra $A(\bar{D})$ and $f$ is equal to $C(\bar{D})$. When $f$ is $C^{1}$ on $\bar{D}$ (i.e., extends to be continuously differentiable on a neighborhood of $\bar{D})$, this result follows from a theorem of John Wermer [Wer]. E. M. Čirka [Č] observed that Wermer's technique yields a more general theorem, from which the above result can be obtained. More recently, Sheldon Axler and Allen Shields [A-S] gave an entirely different proof of the result and suggested generalizing the result to the setting of several complex variables. In particular, they posed the following question: If $f_{1}, \ldots, f_{n}$ are in the polydisc algebra $A\left(\bar{D}^{n}\right)$ (or the ball algebra $A\left(\bar{B}_{n}\right)$ ) and are independent (in some appropriate sense), is the norm-closed algebra generated by $A\left(\bar{D}^{n}\right)$ (or $A\left(\bar{B}_{n}\right)$ ) and the complex conjugates of $f_{1}, \ldots, f_{n}$ equal to $C\left(\bar{D}^{n}\right)$ (or $\left.C\left(\bar{B}_{n}\right)\right)$ ? This paper is devoted to attacking that question.

Section 1 deals with the ball algebra. The main result asserts that if $f_{1}, \ldots$, $f_{n}$ are pluriharmonic on $B_{n}$ (the open unit ball in $\mathbb{C}^{n}$ ) and $C^{1}$ on $\bar{B}_{n}$ (i.e., extend to be continuously differentiable on a neighborhood of $\bar{B}_{n}$ ), and the $n \times n$ matrix $\left(\partial f_{j} / \partial \bar{z}_{k}\right)$ is invertible at every point of $B_{n}$, then the norm-closed algebra generated by the ball algebra $A\left(\bar{B}_{n}\right)$ and $f_{1}, \ldots, f_{n}$ is equal to $C\left(\bar{B}_{n}\right)$. In $\S 2$, extensions of the results of $\S 1$ to more general strictly pseudoconvex domains are presented. In $\S 3$, the situation for the polydisc is discussed briefly. The paper concludes with some open questions.

Received by the editors July 22, 1991.

1991 Mathematics Subject Classification. Primary 32E25, 46J10, 46J15; Secondary 32E20, $32 \mathrm{~F} 15$.

This research was partially supported by NSF grant DMS 90-02904. 
Given a uniform algebra $A$ on a compact space $X$, and continuous functions $f_{1}, \ldots, f_{n}$ on $X$, we will write $A\left[f_{1}, \ldots, f_{n}\right]$ to denote the norm-closed subalgebra of $C(X)$ generated by $A$ and the functions $f_{1}, \ldots, f_{n}$. As usual, $z_{1}, \ldots, z_{n}$ will denote the complex coordinate functions on $\mathbb{C}^{n}$, and the differential operators $D_{j}$ and $\bar{D}_{j}$ will be defined by

$$
D_{j}=\frac{\partial}{\partial z_{j}} \quad \text { and } \quad \bar{D}_{j}=\frac{\partial}{\partial \bar{z}_{j}} .
$$

Some of the material in this paper is from my doctoral dissertation [I], and it is a pleasure to take this opportunity to thank my thesis advisor, Donald Sarason, for his valuable guidance. I am also especially indebted to Barnet Weinstock, who pointed out Theorem 2.5 and its proof, and to David Barrett, whose assistance was crucial in working out Examples 2.6 and 2.7. Finally, I would like to acknowledge my debt to Allen Shields, whose talk in the Functional Analysis Colloquium at Berkeley on November 10, 1986 first interested me in the problems considered here.

\section{THE BALL}

Before looking for conditions under which $n$ functions generate $C\left(\bar{B}_{n}\right)$ when adjoined to the ball algebra $A\left(\bar{B}_{n}\right)$, it seems appropriate to consider the question of whether $C\left(\bar{B}_{n}\right)$ can be generated from the ball algebra by adjoining fewer than $n$ functions. It is not surprising that the answer is negative.

1.1 Theorem. Suppose $k$ and $n$ are positive integers with $k<n$, and $f_{1}, \ldots$, $f_{k}$ are continuous complex-valued functions on $\bar{B}_{n}$. Then $A\left(\bar{B}_{n}\right)\left[f_{1}, \ldots, f_{k}\right] \neq$ $C\left(\bar{B}_{n}\right)$.

Let $S$ denote the unit sphere in $\mathbb{C}^{n}$ and let $A(S)$ denote the uniform algebra on $S$ consisting of the continuous functions on $S$ that are restrictions of elements of the ball algebra. Then the above theorem is clearly a consequence of the following:

1.2 Theorem. Suppose $k$ and $n$ are as above, and $f_{1}, \ldots, f_{k}$ are continuous complex-valued functions on the unit sphere $S$ in $\mathbb{C}^{n}$. Then $A(S)\left[f_{1}, \ldots, f_{k}\right] \neq$ $C(S)$.

We will see in a moment that this theorem follows readily from a result due to Andrew Browder [B]. If $A$ is a unital commutative Banach algebra, a subset $E$ of $A$ is said to be a set (or system) of generators for $A$ if the smallest unital closed subalgebra of $A$ containing $E$ is $A$ itself, or equivalently, if the polynomials in the elements of $E$ form a dense subalgebra of $A$. A theorem of Browder [B] relates the minimum number of elements in a set of generators for a unital commutative Banach algebra to the Cech cohomology of the algebra's maximal ideal space. We will need only the following corollary of this theorem (also due to Browder [B]).

1.3 Corollary. If $S^{N}$ denotes the $N$-sphere (i.e., the set $\left\{x \in \mathbb{R}^{N+1}:|x|=1\right\}$ ), then $C\left(S^{N}\right)$ has no system of fewer than $N+1$ generators.

To see that Theorem 1.2 follows from this result, note that since polynomials in the $n$ variables $z_{1}, \ldots, z_{n}$ are dense in $A(S)$, the set $\left\{z_{1}, \ldots, z_{n}, f_{1}, \ldots\right.$, 
$\left.f_{k}\right\}$ is a system of $n+k$ generators for $A(S)\left[f_{1}, \ldots, f_{k}\right]$, while the preceding result asserts that $C(S)$ has no system of fewer than $2 n$ generators.

Having shown that at least $n$ continuous functions must be adjoined to the ball algebra $A\left(\bar{B}_{n}\right)$ to generate $C\left(\bar{B}_{n}\right)$, we turn to the question of when $n$ continuous functions do indeed generate $C\left(\bar{B}_{n}\right)$ when adjoined to $A\left(\bar{B}_{n}\right)$. The fact that at least $n$ continuous functions are needed can be reformulated as follows: If $f_{1}, \ldots, f_{n}$ are continuous functions on $\bar{B}_{n}$, a necessary condition for the norm-closed algebra $A\left(\bar{B}_{n}\right)\left[f_{1}, \ldots, f_{n}\right]$ generated by $A\left(\bar{B}_{n}\right)$ and the functions $f_{1}, \ldots, f_{n}$ to be equal to $C\left(\bar{B}_{n}\right)$, is that no one of the $f_{j}$ is in the norm-closed algebra generated by $A\left(\bar{B}_{n}\right)$ and the other $f_{j}$ 's. This necessary condition is obviously not sufficient even in the case $n=1$. (For example, take the single function $f_{1}$ to be zero on a nonempty proper open subset of the disc.) However, the result mentioned at the beginning of the introduction shows that in the case $n=1$, the condition is sufficient if we assume that the function being adjoined is harmonic on the open unit disc. One might expect a similar statement to hold for $n>1$. However, the following example shows that this is not the case even if the functions adjoined to the ball algebra are assumed to be complex conjugates of polynomials.

1.4 Example. Take $n=2$ and consider $A\left(\bar{B}_{2}\right)\left[\bar{z}_{1}, \bar{z}_{1} \bar{z}_{2}\right]$. Each level set of $\bar{z}_{1}$ is a disc, and the restriction of any member of $A\left(\bar{B}_{2}\right)\left[\bar{z}_{1}\right]$ to any one of these discs is holomorphic. It follows that $\bar{z}_{1} \bar{z}_{2} \notin A\left(\bar{B}_{2}\right)\left[\bar{z}_{1}\right]$. On the other hand, the restriction of any member of $A\left(\bar{B}_{2}\right)\left[\bar{z}_{1} \bar{z}_{2}\right]$ to the disc $\left\{z_{2}=0,\left|z_{1}\right| \leq 1\right\}$ is holomorphic, so $\bar{z}_{1} \notin A\left(\bar{B}_{2}\right)\left[\bar{z}_{1} \bar{z}_{2}\right]$. Since $\bar{z}_{1}$ and $\bar{z}_{1} \bar{z}_{2}$ both vanish on the disc $\left\{z_{1}=0,\left|z_{2}\right| \leq 1\right\}$, the restriction of each member of $A\left(\bar{B}_{2}\right)\left[\bar{z}_{1}, \bar{z}_{1} \bar{z}_{2}\right]$ to this disc is holomorphic. Thus $A\left(\bar{B}_{2}\right)\left[\bar{z}_{1}, \bar{z}_{1} \bar{z}_{2}\right] \neq C\left(\bar{B}_{2}\right)$. (For a similar example with $n>2$ consider $A\left(\bar{B}_{n}\right)\left[\bar{z}_{1}, \bar{z}_{1} \bar{z}_{2}, \bar{z}_{3}, \ldots, \bar{z}_{n}\right]$.)

In view of the above example, it is natural to look for stronger independence conditions to impose on the functions being adjoined to the ball algebra. The next result will suggest such a condition.

1.5 Theorem. If $f_{1}, \ldots, f_{n}$ are in $A\left(\bar{B}_{n}\right)$ and for some index $l$, the function $\bar{f}_{l}$ is in the norm-closed algebra generated by $A\left(\bar{B}_{n}\right)$ and the functions $\bar{f}_{j}$ for $j \neq l$, then the matrix $\left(D_{k} f_{j}\right)$ is noninvertible at every point of $B_{n}$.

Proof: Without loss of generality we take the case $\bar{f}_{n} \in A\left(\bar{B}_{n}\right)\left[\bar{f}_{1}, \ldots, \bar{f}_{n-1}\right]$. Let $w$ be an arbitrary but fixed point of $B_{n}$. We are to show that the matrix $\left(\left(D_{k} f_{j}\right)(w)\right)$ is not invertible. If the first $n-1$ rows of the matrix $\left(\left(D_{k} f_{j}\right)(w)\right)$ are not independent, then there is nothing to show, so assume that they are independent. Since $n-1$ vectors cannot span an $n$-dimensional vector space, one of the elements of the standard basis for $\mathbb{C}^{n}$ must lie outside the linear span of the first $n-1$ rows of the matrix $\left(\left(D_{k} f_{j}\right)(w)\right)$, and we may assume without loss of generality that it is the vector $(0, \ldots, 0,1)$. Then the matrix

$$
\left(\begin{array}{cccc}
D_{1} f_{1} & \ldots & D_{n-1} f_{1} & D_{n} f_{1} \\
\vdots & \ddots & \vdots & \vdots \\
D_{1} f_{n-1} & \ldots & D_{n-1} f_{n-1} & D_{n} f_{n-1} \\
0 & \ldots & 0 & 1
\end{array}\right)
$$

is invertible at $w$. 
By adding constants to the functions $f_{1}, \ldots, f_{n}$, we can assume without loss of generality that they all vanish at $w$. Now define $F: B_{n} \rightarrow \mathbb{C}^{n}$ by $F(z)=\left(f_{1}(z), \ldots, f_{n-1}(z), z_{n}-w_{n}\right)$. Then $F$ is a holomorphic map, and its (complex) derivative is the linear map induced by the matrix above. Hence the inverse function theorem (for holomorphic maps) shows that there are neighborhoods $W$ of $w$ and $W^{\prime}$ of $F(w) \quad(=0)$ such that $F$ is a one-to-one map of $W$ onto $W^{\prime}$ whose inverse $F^{-1}: W^{\prime} \rightarrow W$ is holomorphic. Now if $\Delta$ is a sufficiently small disc about the origin in $\mathbb{C}$, we can define a holomorphic map $\gamma: \Delta \rightarrow B_{n}$ by $\gamma(z)=F^{-1}(0, \ldots, 0, z)$. Then each of the functions $f_{1} \circ \gamma, \ldots, f_{n-1} \circ \gamma$ is the zero function, and hence so are their complex conjugates $\bar{f}_{1} \circ \gamma, \ldots, \bar{f}_{n-1} \circ \gamma$. Since $\bar{f}_{n} \in A\left(\bar{B}_{n}\right)\left[\bar{f}_{1}, \ldots, \bar{f}_{n-1}\right]$, it follows that $\bar{f}_{n} \circ \gamma$ is holomorphic. Since $f_{n} \circ \gamma$ is obviously holomorphic, we conclude that $f_{n} \circ \gamma$ is constant. Thus the derivative of the complex-valued function defined on $\Delta$ by $z \mapsto\left(\left(f_{1} \circ \gamma\right)(z), \ldots,\left(f_{n} \circ \gamma\right)(z)\right)$ is zero. Since the derivative of $\gamma$ is obviously not zero at the origin, it follows from the chain rule that the matrix $\left(\left(D_{k} f_{j}\right)(w)\right)$ is not invertible.

Reexamination of Example 1.4 reveals that given functions $f_{1}, \ldots, f_{n}$ in the ball algebra, the condition that the matrix $\left(D_{k} f_{j}\right)$ be invertible at some point of $B_{n}$ is not sufficient to guarantee that $A\left(\bar{B}_{n}\right)\left[\bar{f}_{1}, \ldots, \bar{f}_{n}\right]=C\left(\bar{B}_{n}\right)$. In the example, the matrix in question is invertible at some points and not at others. This suggests that we should require invertibility at every point of $B_{n}$. We are thus led to the following question.

1.6 Question. If $f_{1}, \ldots, f_{n}$ are in $A\left(\bar{B}_{n}\right)$ and the matrix $\left(D_{k} f_{j}\right)$ is invertible at every point of $B_{n}$, is $A\left(\bar{B}_{n}\right)\left[\bar{f}_{1}, \ldots, \bar{f}_{n}\right]$ equal to $C\left(\bar{B}_{n}\right)$ ?

This question is still open. In the special case where the functions $f_{1}, \ldots, f_{n}$ are $C^{1}$ on $\bar{B}_{n}$, the answer is affirmative. Explicitly:

1.7 Theorem. If $f_{1}, \ldots, f_{n}$ are in $A\left(\bar{B}_{n}\right)$ and are $C^{1}$ on $\bar{B}_{n}$, and the matrix $\left(D_{k} f_{j}\right)$ is invertible at every point of $B_{n}$, then $A\left(\bar{B}_{n}\right)\left[\bar{f}_{1}, \ldots, \bar{f}_{n}\right]=C\left(\bar{B}_{n}\right)$.

This theorem will be proven below by investigating the maximal sets of antisymmetry for $A\left(\bar{B}_{n}\right)\left[\bar{f}_{1}, \ldots, \bar{f}_{n}\right]$. (A subset $E$ of $\bar{B}_{n}$ is a set of antisymmetry for $A\left(\bar{B}_{n}\right)\left[\bar{f}_{1}, \ldots, \bar{f}_{n}\right]$ if every function in $A\left(\bar{B}_{n}\right)\left[\bar{f}_{1}, \ldots, \bar{f}_{n}\right]$ that is real-valued on $E$ is in fact constant on $E$.)

1.8 Lemma. If $f_{1}, \ldots, f_{n}$ are in $A\left(\bar{B}_{n}\right)$ and the matrix $\left(D_{k} f_{j}\right)$ is invertible at every point of $B_{n}$, then the functions $f_{1}, \ldots, f_{n}$ are constant on each set of antisymmetry for $A\left(\bar{B}_{n}\right)\left[\bar{f}_{1}, \ldots, \bar{f}_{n}\right]$, and moreover each maximal set of antisymmetry is either a single point or is a connected polynomially convex subset of $S$.

Before proving Lemma 1.8 , we establish a very easy sublemma.

1.9 Sublemma. Under the hypotheses of Lemma 1.8, if $L$ is a level set of the function $z \mapsto\left(f_{1}(z), \ldots, f_{n}(z)\right)$, then $L$ is polynomially convex, and the intersection of $L$ with $B_{n}$ is discrete.

Proof. By adding constants to the functions $f_{1}, \ldots, f_{n}$, we can assume without loss of generality that $L$ is the common zero set $Z\left(f_{1}, \ldots, f_{n}\right)$ of the 
function $f_{1}, \ldots, f_{n}$. Since each $f_{j}$ is the uniform limit of polynomials, the set $Z\left(f_{1}, \ldots, f_{n}\right)$ is polynomially convex.

Since the matrix $\left(D_{k} f_{j}\right)$ is assumed invertible at every point of $B_{n}$, the inverse function theorem shows that the map $z \mapsto\left(f_{1}(z), \ldots, f_{n}(z)\right)$ is locally one-to-one on $B_{n}$. Consequently, the intersection of $Z\left(f_{1}, \ldots, f_{n}\right)$ with $B_{n}$ is discrete.

Proof of Lemma 1.8. Since both $f_{j}$ and $\bar{f}_{j}(j=1, \ldots, n)$ are in the algebra $A\left(\bar{B}_{n}\right)\left[\bar{f}_{1}, \ldots, \bar{f}_{n}\right]$, the real and imaginary parts of $f_{j}$ are in the algebra $A\left(\bar{B}_{n}\right)\left[\bar{f}_{1}, \ldots, \bar{f}_{n}\right]$ and hence are constant on each set of antisymmetry for $A\left(\bar{B}_{n}\right)\left[\bar{f}_{1}, \ldots, \bar{f}_{n}\right]$. This establishes the first assertion.

Now let $E$ be a maximal set of antisymmetry for $A\left(\bar{B}_{n}\right)\left[\bar{f}_{1}, \ldots, \bar{f}_{n}\right]$. We have just observed that each $f_{j}$ is constant on $E$, so $E$ is contained in a common level set $L$ of the functions $f_{1}, \ldots, f_{n}$. By the sublemma, $L$ is polynomially convex. Hence the polynomial convex hull $\hat{E}$ of $E$ is contained in $L$. We now show that $\hat{E}$ is a set of antisymmetry for $A\left(\bar{B}_{n}\right)\left[\bar{f}_{1}, \ldots, \bar{f}_{n}\right]$, and hence $\hat{E}=E$, so $E$ is polynomially convex. So suppose $g \in A\left(\bar{B}_{n}\right)\left[\bar{f}_{1}, \ldots, \bar{f}_{n}\right]$ is real valued on $\hat{E}$. Then, in particular, $g$ is real valued on $E$, hence constant on $E$. So without loss of generality $g$ is zero on $E$. Since each $\bar{f}_{j}$ is constant on $\hat{E}$, we see that $g$ can be approximated uniformly on $\hat{E}$ by elements of $A\left(\bar{B}_{n}\right)$, and hence by polynomials. Since the value of a polynomial $p$ at any point of $\hat{E}$ is bounded by the supremum of $p$ over $E$, it follows that $g$ vanishes on $\hat{E}$. Thus $\hat{E}$ is a set of antisymmetry as asserted, so $E$ is polynomially convex.

Now assume, to get a contradiction, that $E$ is not connected. Then there exist disjoint nonempty relatively closed sets $E_{0}$ and $E_{1}$ whose union is $E$. Since $E$ is closed in $\mathbb{C}^{n}$, so are $E_{0}$ and $E_{1}$. Consequently, there are disjoint open sets $U_{0}$ and $U_{1}$ of $\mathbb{C}^{n}$ such that $E_{0} \subset U_{0}$ and $E_{1} \subset U_{1}$. Hence the function $h$ that is 0 on $E_{0}$ and 1 on $E_{1}$ extends to be holomorphic on the neighborhood $U_{0} \cup U_{1}$ of $E$, and therefore, by the Oka-Weil approximation theorem [G, Theorem III.5.1] can be approximated uniformly on $E$ by polynomials. It follows that $h$ is in $A\left(\bar{B}_{n}\right)\left[\bar{f}_{1}, \ldots, \bar{f}_{n}\right] \mid E$ which contradicts the fact that $E$ is a set of antisymmetry for $A\left(\bar{B}_{n}\right)\left[\bar{f}_{1}, \ldots, \bar{f}_{n}\right]$. Therefore, $E$ must be connected.

Recalling that $E$ is contained in the common level set $L$ of $f_{1}, \ldots, f_{n}$, and that the intersection of $L$ with $B_{n}$ is discrete by the sublemma, we see that the connectedness of $E$ implies that either $E$ consists of a single point or is contained in $S$.

If $X$ is a compact subset of $\mathbb{C}^{n}$, we denote by $P(X)$ the uniform algebra of continuous functions on $X$ that can be approximated uniformly by polynomials in $z_{1}, \ldots, z_{n}$. Combining Lemma 1.8 with the Bishop antisymmetric decomposition we obtain the following:

1.10 Corollary. Suppose $f_{1}, \ldots, f_{n}$ are in $A\left(\bar{B}_{n}\right)$ and the matrix $\left(D_{k} f_{j}\right)$ is invertible at every point of $B_{n}$. Let $\left\{X_{\alpha}\right\}$ be the decomposition of $S$ into the common level sets of the functions $f_{1}, \ldots, f_{n}$. Then $A\left(\bar{B}_{n}\right)\left[\bar{f}_{1}, \ldots, \bar{f}_{n}\right]=$ $C\left(\bar{B}_{n}\right)$ if and only if $P\left(X_{\alpha}\right)=C\left(X_{\alpha}\right)$ for each $\alpha$.

Unfortunately it is generally very difficult to tell whether a compact set $X$ 
in $\mathbb{C}^{n}(n>1)$ has the property that $P(X)=C(X)$. However, the next theorem shows that if the functions $f_{1}, \ldots, f_{n}$ are $C^{1}$ on $\bar{B}_{n}$, then the sets $X_{\alpha}$ in the above corollary are in fact interpolation sets for the ball algebra (i.e., $\left.A\left(\bar{B}_{n}\right) \mid X_{\alpha}=C\left(X_{\alpha}\right)\right)$.

1.11 Theorem. If $f_{1}, \ldots, f_{n}$ are in $A\left(\bar{B}_{n}\right)$ and $C^{1}$ on $\bar{B}_{n}$, and the matrix $\left(D_{k} f_{j}\right)$ is invertible at every point of $B_{n}$, then each subset of $S$ on which all the $f_{j}$ are constant is an interpolation set for the ball algebra.

Proof: Let $E$ be a subset of $S$ on which all the $f_{j}$ are constant. Then $E$ is contained in a level set $L$ of the function $F$ defined on $\bar{B}_{n}$ by $F(z)=$ $\left(f_{1}(z), \ldots, f_{n}(z)\right)$. The inverse function theorem shows that any point of $L$ where the complex Jacobian $J F$ of $F$ does not vanish is an isolated point of $L$. Consequently, $L$ is the union of a discrete set with a subset of the zero set of $J F$. Hence the same is true of $E$. The function $J F$ is easily seen to be in $A\left(\bar{B}_{n}\right)$, and does not vanish on $B_{n}$ (since invertibility of $\left(D_{k} f_{j}\right)$ is equivalent to the nonvanishing of $J F$ ). It is a standard theorem [R, Theorem 10.1.2] that a subset of $S$ is an interpolation set for the ball algebra if and only if it is the zero set of some member of the ball algebra. Thus, in particular, the zero set of $J F$ is an interpolation set for the ball algebra. Since any discrete subset of the ball is countable, and a one-point set is obviously an interpolation set, we conclude that $E$ is a countable union of interpolation sets for the ball algebra. It follows [R, 10.1.5] that $E$ is an interpolation set for the ball algebra.

The above proof goes through without substantial change if the hypothesis that the functions $f_{1}, \ldots, f_{n}$ be $C^{1}$ on $\bar{B}_{n}$ is replaced by the seemingly weaker hypothesis that the derivatives of the functions $f_{1}, \ldots, f_{n}$ be uniformly continuous on $B_{n}$. However, this observation does not lead to a generalization, for if $\Omega$ is an open set in $\mathbb{R}^{k}$ with $C^{1}$ boundary, and $f: \bar{\Omega} \rightarrow \mathbb{R}$ is a continuous function with a uniformly continuous derivative on $\Omega$, then $f$ has a $C^{1}$ extension to $\mathbb{R}^{k}$. To see this, note that by a partition of unity argument, it suffices to consider the special case where $\Omega$ is the open upper half-space $\left\{x_{k}>0\right\}$. To obtain the desired extension $\tilde{f}$ in this case, assume without loss of generality that $f$ is zero on $\left\{x_{k}=0\right\}$ and define $\tilde{f}: \mathbb{R}^{k} \rightarrow \mathbb{R}$ by

$$
\tilde{f}\left(x_{1}, \ldots, x_{k}\right)=\left\{\begin{array}{cc}
f\left(x_{1}, \ldots, x_{k}\right) & \text { if } x_{k} \geq 0, \\
-f\left(x_{1}, \ldots, x_{k-1},-x_{k}\right) & \text { if } x_{k} \leq 0 .
\end{array}\right.
$$

Note that Theorem 1.7 is an immediate consequence of Theorem 1.11 and Corollary 1.10. The next lemma will enable us to extend Theorem 1.7 to pluriharmonic functions by reducing to the case already considered.

1.12 Lemma. If $f$ is a real-valued function in $C\left(\bar{B}_{n}\right)$ that is pluriharmonic on $B_{n}$ and $C^{1}$ on $\bar{B}_{n}$, then there is a $C^{1}$ real-valued function $f^{*}$ on $\bar{B}_{n}$ such that $f+i f^{*}$ is in $A\left(\bar{B}_{n}\right)$.

Proof: It is well known that every real-valued pluriharmonic function on $B_{n}$ is the real part of some holomorphic function on $B_{n}$. Thus there is a function $g$ on $B_{n}$ such that $f+i g$ is holomorphic on $B_{n}$. In view of the remarks about $C^{1}$ functions made above, the Cauchy-Riemann equations show that $g$ has a $C^{1}$ extension to $\bar{B}_{n}$. Letting $f^{*}$ be that extension we obtain the lemma. 
1.13 Theorem. If $f_{1}, \ldots, f_{n}$ in $C\left(\bar{B}_{n}\right)$ are pluriharmonic on $B_{n}$ and $C^{1}$ on $\bar{B}_{n}$, and the matrix $\left(\bar{D}_{k} f_{j}\right)$ is invertible at every point of $B_{n}$, then

$$
A\left(\bar{B}_{n}\right)\left[f_{1}, \ldots, f_{n}\right]=C\left(\bar{B}_{n}\right) .
$$

Proof. Let $u_{j}$ and $v_{j}$ denote the real and imaginary parts of $f_{j}$, respectively. Let $g_{j}=\left(u_{j}+v_{j}^{*}\right)+i\left(u_{j}^{*}-v_{j}\right)$. Then clearly $g_{j}=\left(u_{j}+i u_{j}^{*}\right)-i\left(v_{j}+i v_{j}^{*}\right)$ so $g_{j}$ is in $A\left(\bar{B}_{n}\right)$. Moreover, the imaginary part of $g_{j}$ can be written as $-i\left(u_{j}+i u_{j}^{*}\right)+i\left(u_{j}+i v_{j}\right)$ and hence is clearly in $A\left(\bar{B}_{n}\right)\left[f_{1}, \ldots, f_{n}\right]$. Hence $\bar{g}_{j}$ is in $A\left(\bar{B}_{n}\right)\left[f_{1}, \ldots, f_{n}\right]$. In addition, it is clear that $g_{j}$ is $C^{1}$ on $\bar{B}_{n}$. Thus the desired conclusion will follow from Theorem 1.7 as soon as we show that the matrix $\left(D_{k} g_{j}\right)$ is invertible at every point of $B_{n}$. Now note that

$$
\begin{aligned}
D_{k} g_{j} & =D_{k}\left[\left(u_{j}+i u_{j}^{*}\right)-i\left(v_{j}+i v_{j}^{*}\right)\right] \\
& =2 D_{k}\left(u_{j}-i v_{j}\right) \quad \text { (by the Cauchy-Riemann equations) } \\
& =\overline{2 \bar{D}_{k}\left(u_{j}+i v_{j}\right)} \\
& =\overline{2 \bar{D}_{k} f_{j}},
\end{aligned}
$$

so the assumed invertibility of $\left(\bar{D}_{k} f_{j}\right)$ implies the needed invertibility of $\left(D_{k} g_{j}\right)$.

Recall from the introduction, that if $f$ is in $C(\bar{D})$ and is harmonic but not holomorphic on $D$, then $A(\bar{D})[f]=C(\bar{D})$. Since the $\bar{z}$-derivative of such a function may have zeros, it follows that the condition in Theorem 1.13 that the matrix $\left(\bar{D}_{k} f_{j}\right)$ be invertible at every point of $B_{n}$ is not a necessary condition, at least when $n=1$. It follows from the next result that the same is true when $n>1$ also.

1.14 Theorem. If $f_{1}, \ldots, f_{n}$ are functions in $C\left(\bar{B}_{n}\right)$ such that each $f_{j}$ depends only on $z_{j}$, and the functions $f_{1}, \ldots, f_{n}$ are harmonic but not holomorphic on $\bar{B}_{n}$, then $A\left(\bar{B}_{n}\right)\left[f_{1}, \ldots, f_{n}\right]=C\left(\bar{B}_{n}\right)$.

Proof: It is immediate from the result about the disc algebra recalled above that $A\left(\bar{B}_{n}\right)\left[f_{j}\right]$ contains every function in $C\left(\bar{B}_{n}\right)$ that depends only on $z_{j}$. Thus $A\left(\bar{B}_{n}\right)\left[f_{1}, \ldots, f_{n}\right]$ contains every function in $C\left(\bar{B}_{n}\right)$ that depends on only one variable, and the desired conclusion follows by applying the Stone-Weierstrass theorem.

\section{STRICTLY PSEUDOCONVEX DOMAINS}

This section is devoted to extending the results of $\S 1$ to more general domains. We first observe that Theorem 1.1 continues to hold if the ball is replaced by an arbitrary open set in $\mathbb{C}^{n}$. Explicitly:

2.1 Theorem. Suppose that $\Omega$ is an open set in $\mathbb{C}^{n}$, that $k$ is a positive integer strictly less than $n$, and that $f_{1}, \ldots, f_{k}$ are continuous complex-valued functions on $\bar{\Omega}$. Then $A(\bar{\Omega})\left[f_{1}, \ldots, f_{k}\right] \neq C(\bar{\Omega})$.

Proof. Let $w$ be a point in $\Omega$, and let $B$ be an open ball centered at $w$ and contained in $\Omega$. Obviously it suffices to show that $A(\bar{\Omega})\left[f_{1}, \ldots, f_{k}\right] \mid \bar{B} \neq$ $C(\bar{B})$. Hence since it is clear that $A(\bar{\Omega})\left[f_{1}, \ldots, f_{k}\right] \mid \bar{B} \subset A(\bar{B})\left[f_{1}\left|\bar{B}, \ldots, f_{k}\right| \bar{B}\right]$ the result follows immediately from Theorem 1.1. 
Obviously an argument rather similar to the one just given shows that Theorem 1.5 continues to hold with the ball replaced by an arbitrary open set in $\mathbb{C}^{n}$.

The following theorem extends Theorem 1.7 to arbitrary strictly pseudoconvex domains with $C^{2}$-boundary.

2.2 Theorem. Suppose $\Omega$ is a strictly pseudoconvex domain in $\mathbb{C}^{n}$ with $C^{2}$ boundary, $f_{1}, \ldots, f_{n}$ are in $A(\bar{\Omega})$ and are $C^{1}$ on $\bar{\Omega}$, and the matrix $\left(D_{k} f_{j}\right)$ is invertible at every point of $\Omega$. Then $A(\bar{\Omega})\left[\bar{f}_{1}, \ldots, \bar{f}_{n}\right]=C(\bar{\Omega})$.

The proof of Theorem 1.7 given in $\S 1$ does not carry over to this more general situation without significant modification. This is because Sublemma 1.9 relies on the polynomial convexity of the closed ball, and the closure of a strictly pseudoconvex domain need not be polynomially convex. (An example of a strictly pseudoconvex domain whose closure is not polynomially convex is given at the end of this section.) The proof given below is actually more direct than the proof of Theorem 1.7 given in $\S 1$. However, this more direct approach does not yield Corollary 1.10. Since that result may lead to the answer to Question 1.6, the extra work done in $\S 1$ seems justified.

Proof of Theorem 2.2. By the Bishop antisymmetric decomposition it suffices to show that each maximal set of antisymmetry for $A(\bar{\Omega})\left[\bar{f}_{1}, \ldots, \bar{f}_{n}\right]$ is an interpolation set for $A(\bar{\Omega})\left[\bar{f}_{1}, \ldots, \bar{f}_{n}\right]$. So let $E$ be a maximal set of antisymmetry for $A(\bar{\Omega})\left[\bar{f}_{1}, \ldots, \bar{f}_{n}\right]$. Then $E$ is closed in $\bar{\Omega}$. Since the real and imaginary parts of each $f_{j}$ are in $A(\bar{\Omega})\left[\bar{f}_{1}, \ldots, \bar{f}_{n}\right]$, each $f_{j}$ must be constant on $E$. Hence the inverse function theorem shows that every point of $E$ where the determinant of $\left(D_{k} f_{j}\right)$ does not vanish is an isolated point of $E$. In particular, the intersection of $E$ with $\Omega$ is discrete. Hence either $E$ is finite, or $E$ intersects $\partial \Omega$. If $E$ is finite, then obviously $E$ is an interpolation set. So assume from now on that $E$ intersects $\partial \Omega$.

Since every point of $E$ where the determinant of the matrix $\left(D_{k} f_{j}\right)$ does not vanish is an isolated point of $E$, we see that $E \cap \partial \Omega$ is the union of a discrete set with a subset of the zero set of the function $\operatorname{det}\left(D_{k} f_{j}\right)$. Since the function $\operatorname{det}\left(D_{k} f_{j}\right)$ is easily seen to be in $A(\bar{\Omega})$, and does not vanish on $\Omega$, its zero set is an interpolation set for $A(\bar{\Omega})$ (by Theorem 1.1 in [Wei1]). It follows that $E \cap \partial \Omega$ is a countable union of interpolation sets for $A(\bar{\Omega})$. Applying Theorem 1.1 in [Weil] again, we conclude that $E \cap \partial \Omega$ is an interpolation set for $A(\bar{\Omega})$, or equivalently that it is the zero set of some function in $A(\bar{\Omega})$. Now it suffices to show that $E \cap \Omega$ is empty.

Assume to get a contradiction that $E \cap \Omega$ is nonempty. Since $E \cap \Omega$ is discrete, it is countable, so denote the points of $E \cap \Omega$ by $\alpha_{1}, \alpha_{2}, \ldots$. We will show that the function that is 1 at $\alpha_{1}$ and 0 on $(E \cap \partial \Omega) \cup\left\{\alpha_{2}, \alpha_{3}, \ldots\right\}$ is in $A(\bar{\Omega})\left[\bar{f}_{1}, \ldots, \bar{f}_{n}\right] \mid E$, a contradiction since $E$ is a set of antisymmetry for $A(\bar{\Omega})\left[\bar{f}_{1}, \ldots, \bar{f}_{n}\right]$.

Choose a function $f$ in $A(\bar{\Omega})$ with $E \cap \partial \Omega$ as its zero set, and assume without loss of generality that $f\left(\alpha_{1}\right)=1$. Note that if $\alpha_{1}$ is the only point in $E \cap \Omega$, then the function $f$ yields the desired contradiction at once, so assume $E \cap \Omega$ consists of more than one point. Then since the $\alpha_{j}$ can accumulate only on $E \cap \partial \Omega$, we have that $\left|f\left(\alpha_{j}\right)\right| \leq \frac{1}{2}$ for all but finitely many $\alpha_{j}$. (Of course 
if there are only finitely many $\alpha_{j}$, then this is vacuously true.) Choose $k$ such that $\left|f\left(\alpha_{j}\right)\right| \leq \frac{1}{2}$ for all $j>k$. (If there are only finitely many $\alpha_{j}$, just set $k$ equal to the number of $\alpha_{j}$.) Since for each $j \neq 1$ the points $\alpha_{1}$ and $\alpha_{j}$ differ in some coordinate, we can let $m_{j}$ denote some coordinate in which they differ. Let

$$
g=\frac{z_{m_{2}}-z_{m_{2}}\left(\alpha_{2}\right)}{z_{m_{2}}\left(\alpha_{1}\right)-z_{m_{2}}\left(\alpha_{2}\right)} \cdot \frac{z_{m_{3}}-z_{m_{3}}\left(\alpha_{3}\right)}{z_{m_{3}}\left(\alpha_{1}\right)-z_{m_{3}}\left(\alpha_{3}\right)} \cdots \frac{z_{m_{k}}-z_{m_{k}}\left(\alpha_{k}\right)}{z_{m_{k}}\left(\alpha_{1}\right)-z_{m_{k}}\left(\alpha_{k}\right)}
$$

and let $C$ be the supremum of $|g|$ over $\bar{\Omega}$. Now fix $\varepsilon>0$, and choose a positive integer $N$ large enough that $1 / 2^{N}<\varepsilon / C$. Let $F=g \cdot f^{N}$. Then $F$ is in $A(\bar{\Omega}), F\left(\alpha_{1}\right)=1$, and $|F|<\varepsilon$ on $(E \cap \partial \Omega) \cup\left\{\alpha_{2}, \alpha_{3}, \ldots\right\}$. Since $\varepsilon>0$ was arbitrary, it follows that the function that is 1 at $\alpha_{1}$ and 0 on $(E \cap \partial \Omega) \cup\left\{\alpha_{2}, \alpha_{3}, \ldots\right\}$ is in $A(\bar{\Omega})\left[\bar{f}_{1}, \ldots, \bar{f}_{n}\right] \mid E$. We have arrived at the desired contradiction and the proof is complete.

It is well known that every real-valued pluriharmonic function on a simply connected domain is the real part of some holomorphic function on the domain. Thus in the special case when $\Omega$ is simply connected, Theorem 2.2 can be extended to pluriharmonic functions in the same way that Theorem 1.13 was obtained from Theorem 1.7. Explicitly we obtain the following result.

2.3 Theorem. Suppose $\Omega$ is a simply connected strictly pseudoconvex domain in $\mathbb{C}^{n}$ with $C^{2}$-boundary, $f_{1}, \ldots, f_{n}$ in $C(\bar{\Omega})$ are pluriharmonic on $\Omega$ and $C^{1}$ on $\bar{\Omega}$, and the matrix $\left(\bar{D}_{k} f_{j}\right)$ is invertible at every point of $\Omega$. Then $A(\bar{\Omega})\left[f_{1}, \ldots, f_{n}\right]=C(\bar{\Omega})$.

There is another approach which yields the conclusion of Theorem 2.3 under somewhat different hypotheses. This approach depends on the following lemma which is a special case of a result of Čirka [Č].

2.4 Lemma. Suppose $\Omega$ is a bounded open set in $\mathbb{C}^{n}$ with polynomially convex closure and $f_{1}, \ldots, f_{m}$ in $C(\bar{\Omega})$ are pluriharmonic on $\Omega$ and $C^{2}$ on $\bar{\Omega}$. Then the set $\left\{\left(z, f_{1}(z), \ldots, f_{m}(z)\right): z \in \bar{\Omega}\right\}$ is polynomially convex in $\mathbb{C}^{n+m}$.

As mentioned in the introduction, the following theorem and its proof were pointed out to me by Barnet Weinstock.

2.5 Theorem. Suppose $\Omega$ is a strictly pseudoconvex domain in $\mathbb{C}^{n}$ with $C^{2}$-boundary and polynomially convex closure. Suppose also that $f_{1}, \ldots, f_{n}$ in $C(\bar{\Omega})$ are pluriharmonic on $\Omega$ and $C^{2}$ on $\bar{\Omega}$, and the matrix $\left(\bar{D}_{k} f_{j}\right)$ is invertible at every point of $\Omega$. Then $A(\bar{\Omega})\left[f_{1}, \ldots, f_{n}\right]=C(\bar{\Omega})$.

Note that the hypotheses on $\Omega$ are somewhat different from those in Theorem 2.3 , and that the differentiablity condition on the functions $f_{1}, \ldots, f_{n}$ is slightly more stringent than that in Theorem 2.3 .

Proof. Let $Y=\left\{\left(z, f_{1}(z), \ldots, f_{n}(z)\right): z \in \bar{\Omega}\right\}$ and let $E=\left\{z \in \bar{\Omega}:\left(\bar{D}_{k} f_{j}\right)\right.$ is noninvertible at $z\}$. By Lemma $2.4, Y$ is polynomially convex. Every function in $A(\bar{\Omega})$ can be approximated uniformly by functions holomorphic on a neighborhood of $\bar{\Omega}[\mathrm{R}-\mathrm{S}$, Theorem 5.10], and hence (in view of the OkaWeil approximation theorem) by polynomials in the $n$ variables $z_{1}, \ldots, z_{n}$. Now by a theorem of Weinstock [Wei2], the algebra $A(\bar{\Omega})\left[f_{1}, \ldots, f_{n}\right]$ consists of those continuous functions on $\bar{\Omega}$ that agree with some element of 
$A(\bar{\Omega})\left[f_{1}, \ldots, f_{n}\right]$ on $E$. Thus it suffices to show that $E$ is an interpolation set for $A(\bar{\Omega})$.

It follows from the hypotheses on the functions $f_{1}, \ldots, f_{n}$ that the complex conjugate of the function $\operatorname{det}\left(\bar{D}_{k} f_{j}\right)$ is in $A(\bar{\Omega})$. Thus $E$ is a zero-set for $A(\bar{\Omega})$ and hence (by Theorem 1.1 in [Wei1]) an interpolation set for $A(\bar{\Omega})$.

The hypotheses on $\Omega$ in Theorems 2.3 and 2.5 are incomparable, i.e., neither set of hypotheses implies the other. We conclude this section by giving examples that illustrate this.

2.6 Example. A strictly pseudoconvex domain with smooth boundary and polynomially convex closure need not be simply connected.

Define $\rho: \mathbb{C}^{2} \rightarrow \mathbb{C}$ by

$$
\rho=\left(z_{1} z_{2}-1\right) \overline{\left(z_{1} z_{2}-1\right)}+\left(\operatorname{Re} z_{1}-\operatorname{Re} z_{2}\right)^{2}+\left(\operatorname{Im} z_{1}+\operatorname{Im} z_{2}\right)^{2} .
$$

Then routine computations show that $\left(\bar{D}_{1} \rho, \bar{D}_{2} \rho\right)$ vanishes only on the circle $\{\rho=0\}$ and at the origin, and that the complex Hessian of $\rho$ is strictly positive definite everywhere so $\rho$ is strictly plurisubharmonic. Therefore, the set $\{\rho<r\}$ is strictly pseudoconvex with smooth boundary for $0<r<1$. Let $K=\overline{\{\rho<r\}}(=\{\rho \leq r\})$. Since $\rho$ is strictly plurisubharmonic, it is obvious that the plurisubharmonic convex hull $\hat{K}_{\mathbb{C}^{2}}^{p}$ of $K$ in $\mathbb{C}^{2}$ is just $K$. Hence $K$ is polynomially convex (since $\hat{K}=\hat{K}_{\mathbb{C}^{2}}^{p}$ by Theorem 4.3.4 in [H]). To see that $\{\rho<r\}$ is not simply connected, note that the circle $\left\{\left(e^{i \theta}, e^{-i \theta}\right): 0 \leq \theta \leq 2 \pi\right\}$ lies in $\{\rho<r\}$ but is not null homotopic there, since $\{\rho<r\}$ is contained in $\left\{\left(z_{1}, z_{2}\right) \in \mathbb{C}^{2}: z_{1} \neq 0\right\}$.

2.7 Example. A simply connected strictly pseudoconvex domain with smooth boundary need not have polynomially convex closure.

Let $h$ be a smooth real-valued function on the plane such that

$$
\operatorname{supp} h \subset\left\{|z|^{2} \leq \frac{1}{4}\right\}, \quad \sup |h|<1, \quad h(0)=0,
$$

and

$$
\frac{\partial h}{\partial \bar{z}}(0) \neq 0 \text {. }
$$

Let $x_{j}$ and $y_{j}$ denote respectively the real and imaginary parts of the complex coordinate function $z_{j}$, and define functions $f_{1}, \ldots, f_{4}: \mathbb{C}^{3} \rightarrow \mathbb{R}$ by

$$
\begin{aligned}
& f_{1}=x_{1}^{2}+y_{1}^{2}+x_{2}^{2}+x_{3}^{2}-1, \quad f_{2}=y_{2}, \\
& f_{3}=y_{3}, \quad f_{4}=h\left(x_{1}+i y_{1}\right)-x_{2}-x_{3} .
\end{aligned}
$$

Finally, let $\rho=f_{1}^{2}+f_{2}^{2}+f_{3}^{2}+f_{4}^{2}$. We will show that for $r>0$ sufficiently small, the set $\{\rho<r\}$ in $\mathbb{C}^{3}$ is a simply connected strictly pseudoconvex domain with smooth boundary whose closure is not polynomially convex.

First note that 0 is a regular value of the map $\left(f_{1}, \ldots, f_{4}\right): \mathbb{C}^{3}\left(=\mathbb{R}^{6}\right) \rightarrow \mathbb{R}^{4}$. (To see this, compute the Jacobian matrix and observe that it has maximal rank whenever $x_{2} \neq x_{3}$ and also whenever $x_{1}^{2}+y_{1}^{2}>\frac{1}{4}$. Then show that no point in the preimage of 0 can simultaneously satisfy both $x_{2}=x_{3}$ and $x_{1}^{2}+y_{1}^{2} \leq \frac{1}{4}$.) It follows that $\operatorname{grad} \rho \neq 0$ on the set $\{0<\rho<\varepsilon\}$ for $\varepsilon$ small enough, and hence the set $\{\rho<r\}$ has smooth boundary for $r$ small. Moreover, it also 
follows that for $r$ small, $\{\rho<r\}$ is homeomorphic to $\{\rho=0\} \times B$, where $B$ is an open ball in $\mathbb{R}^{4}$. The reader can verify that $\{\rho=0\}$ is homeomorphic to the two-sphere $S^{2}$. Hence for $r$ small, $\{\rho<r\}$ is homeomorphic to $S^{2} \times B$, and thus is simply connected.

The closure of $\{\rho<r\}$, i.e., the set $\{\rho \leq r\}$, is not polynomially convex when $r<1$, because then the circle $\left\{\left|z_{1}\right|=1, z_{2}=z_{3}=0\right\}$ is contained in $\{\rho \leq r\}$ but the point $(0,0,0)$ is not.

All that remains to be shown is that $\{\rho<r\}$ is strictly pseudoconvex for $r$ small. Thus it suffices to show that the matrix $\left(D_{l} \bar{D}_{k} \rho\right)$ is positive definite on $\{\rho=0\}$ (since the matrix will then be positive definite on a neighborhood of $\{\rho=0\})$. It is easily verified that on $\{\rho=0\}$ we have

$$
\begin{aligned}
D_{l} \bar{D}_{k} \rho & =2\left[\left(D_{l} f_{1}\right)\left(\bar{D}_{k} f_{1}\right)+\cdots+\left(D_{l} f_{4}\right)\left(\bar{D}_{k} f_{4}\right)\right] \\
& =2\left(D_{l} f_{1}, \ldots, D_{l} f_{4}\right) \cdot\left(D_{k} f_{1}, \ldots, D_{k} f_{4}\right) .
\end{aligned}
$$

Routine computation shows that on $\{\rho=0\}$ the vectors $\left(D_{1} f_{1}, \ldots, D_{1} f_{4}\right)$, $\left(D_{2} f_{1}, \ldots, D_{2} f_{4}\right)$, and $\left(D_{3} f_{1}, \ldots, D_{3} f_{4}\right)$ are linearly independent in $\mathbb{C}^{4}$. Now observe that when the matrix for the restriction of the ordinary Hermitian dot product on $\mathbb{C}^{4}$ to

$$
\operatorname{span}\left\{\left(D_{1} f_{1}, \ldots, D_{1} f_{4}\right),\left(D_{2} f_{1}, \ldots, D_{2} f_{4}\right),\left(D_{3} f_{1}, \ldots, D_{3} f_{4}\right)\right\}
$$

is written with respect to the basis $\left\{\left(D_{1} f_{1}, \ldots, D_{1} f_{4}\right),\left(D_{2} f_{1}, \ldots, D_{2} f_{4}\right)\right.$, $\left.\left(D_{3} f_{1}, \ldots, D_{3} f_{4}\right)\right\}$, the resulting matrix is, by $(*)$, simply $\frac{1}{2}\left(D_{l} \bar{D}_{k} \rho\right)$. Hence we conclude that the matrix $\left(D_{l} \bar{D}_{k} \rho\right)$ is positive definite on $\{\rho=0\}$, as desired.

\section{THE POLYDISC}

Theorem 2.1 shows that $C\left(\bar{D}^{n}\right)$ cannot be generated from the polydisc algebra $A\left(\bar{D}^{n}\right)$ by adjoining fewer than $n$ functions. However, since the polydisc is not strictly pseudoconvex, most of the results of $\S 2$ do not apply to the polydisc. In fact, the following example shows that Theorem 2.2 fails for the polydisc, even if the functions $f_{1}, \ldots, f_{n}$ are required to be polynomials.

3.1 Example. Take $n=2$ and let $f_{1}=\left(z_{1}-1\right)^{2}$ and $f_{2}=z_{2}\left(z_{1}-1\right)$. Then the matrix $\left(D_{k} f_{j}\right)$ is

$$
\left(\begin{array}{cc}
2\left(z_{1}-1\right) & 0 \\
z_{2} & z_{1}-1
\end{array}\right)
$$

which is invertible on $D^{n}$. However, since $f_{1}$ and $f_{2}$ both vanish on the set $\left\{z_{1}=1\right\}$, every function in $A\left(\bar{D}^{n}\right)\left[\bar{f}_{1}, \bar{f}_{2}\right]$ is holomorphic on the disc $\left\{z_{1}=1,\left|z_{2}\right| \leq 1\right\}$. Thus $A\left(\bar{D}^{n}\right)\left[\bar{f}_{1}, \bar{f}_{2}\right] \neq C\left(\bar{D}^{n}\right)$.

However, not all is lost. Although we can see from the above example that Theorem 1.11 fails if we replace $B_{n}$ by $D^{n}$ and $S$ by $\partial D^{n}$, using theorems from $\S 21$ of [S], the proof of Theorem 1.11 can be adapted to yield the following weaker analogue of Theorem 1.11. 
3.2 Theorem. If $f_{1}, \ldots, f_{n}$ are in $A\left(\bar{D}^{n}\right)$ and $C^{1}$ on $\bar{D}^{n}$, and the matrix $\left(D_{k} f_{j}\right)$ is invertible at every point of $\bar{D}^{n}$ not on the torus $T^{n}=\left\{\left(z_{1}, \ldots, z_{n}\right) \in\right.$ $\mathbb{C}^{n}:\left|z_{j}\right|=1$ for $\left.1 \leq j \leq n\right\}$, then each subset of $T^{n}$ on which all the $f_{j}$ are constant is an interpolation set for the polydisc algebra.

The following analogues of Theorems 1.7 and 1.13 can also be obtained by making minor modifications to the material in $\S 1$.

3.3 Theorem. If $f_{1}, \ldots, f_{n}$ satisfy the hypotheses of Theorem 3.2, then $A\left(\bar{D}^{n}\right)\left[\bar{f}_{1}, \ldots, \bar{f}_{n}\right]=C\left(\bar{D}^{n}\right)$.

3.4 Theorem. If $f_{1}, \ldots, f_{n}$ in $C\left(\bar{D}^{n}\right)$ are pluriharmonic on $D^{n}$ and $C^{1}$ on $\bar{D}^{n}$, and the matrix $\left(\bar{D}_{k} f_{j}\right)$ is invertible at every point of $\bar{D}^{n} \backslash T^{n}$, then $A\left(\bar{D}^{n}\right)\left[f_{1}, \ldots, f_{n}\right]=C\left(\bar{D}^{n}\right)$.

Finally, note that the proof of Theorem 1.14 remains valid when the ball is replaced by the polydisc.

\section{OPEN QUESTIONS}

4.1. Does Theorem 1.7 continue to hold if the hypothesis that the functions $f_{1}, \ldots, f_{n}$ are $C^{1}$ on $\bar{B}_{n}$ is dropped? (This is a restatement of Question 1.6.) A similar question could also be asked with Theorem 1.13, 2.2, 2.3, or 2.5 in place of Theorem 1.7.

4.2. If $f_{1}, \ldots, f_{n}$ satisfy the conditions in Question 1.6, and $X$ is the subset of $S$ where $f_{1}, \ldots, f_{n}$ all vanish, does $P(X)=C(X)$ ? In view of Corollary 1.10 , this question is equivalent to the above question about Theorem 1.7.

4.3. With $f_{1}, \ldots, f_{n}$ and $X$ as in the preceding question, is $X$ an interpolation set for the ball algebra? (In other words, can the hypothesis that the functions $f_{1}, \ldots, f_{n}$ are $C^{1}$ on $\bar{B}_{n}$ be dropped from Theorem 1.11?) An affirmative answer would of course yield affirmative answers to the two questions above.

4.4. Can Theorems 2.3 and 2.5 be united in some way? More specifically, can the hypothesis that $\Omega$ is simply connected be dropped from Theorem 2.3 , or can the hypothesis that $\Omega$ has polynomially convex closure be dropped from Theorem 2.5?

Note added in proof. The author has obtained an affirmative answer to Question 4.4.

4.5. The question of whether the results of this paper can be extended to the case of $m$ functions of $n$ variables with $m>n$ was suggested to me by Warren Wogan. In particular, if $f_{1}, \ldots, f_{m}$ are in $A\left(\bar{B}_{n}\right)$ (with $m>n$ ) and are $C^{1}$ on $\bar{B}_{n}$, and the matrix $\left(D_{k} f_{j}\right)$ is of maximal rank at every point of $B_{n}$, is $A\left(\bar{B}_{n}\right)\left[\bar{f}_{1}, \ldots, \bar{f}_{m}\right]$ equal to $C\left(\bar{B}_{n}\right)$ ?

\section{REFERENCES}

[A-S] S. Axler and A. Shields, Algebras generated by analytic and harmonic functions, Indiana Univ. Math. J. 36 (1987), 631-638.

[B] A. Browder, Cohomology of maximal ideal spaces, Bull. Amer. Math. Soc. 67 (1961), 515516. 
[Č] E. M. Čirka, Approximation by holomorphic functions on smooth manifolds in $\mathbb{C}^{n}$, Mat. Sb. 78 (1969), 101-123; English transl., Math. USSR Sb. 7 (1969), 95-114.

[G] T. W. Gamelin, Uniform algebras, 2nd ed., Chelsea, New York, 1984.

[H] L. Hörmander, An introduction to complex analysis in several variables, 2nd ed., NorthHolland, 1979.

[I] A. J. Izzo, Uniform algebras generated by holomorphic and harmonic functions of one and several complex variables, Ph.D. Thesis, Univ. of California at Berkeley, 1989.

[R-S] R. M. Range and Y. T. Sui, Uniform estimates for the $\bar{\partial}$-equation on domains with piecewise smooth strictly pseudoconvex boundaries, Math. Ann. 206 (1973), 325-354.

[R] W. Rudin, Function theory in the unit ball of $\mathbb{C}^{n}$, Springer-Verlag, New York, 1980.

[S] E. L. Stout, The theory of uniform algebras, Bogden \& Quigley, New York, 1971.

[Wei1] B. M. Weinstock, Zero-sets of continuous holomorphic functions on the boundary of a strongly pseudoconvex domain, J. London Math. Soc. 18 (1978), 484-488.

[Wei2] B. M. Weinstock, Uniform approximation on the graph of a smooth map in $\mathbf{C}^{n}$, Canad. J. Math. 17 (1980), 1390-1396.

[Wer] J. Wermer, Polynomially convex disks, Math. Ann. 158 (1965), 6-10.

Department of Mathematics, University of Michigan, ANn Arbor, Michigan 48109 02912

Current address: Department of Mathematics, Brown University, Providence, Rhode Island

E-mail address: izzo@gauss.math.brown.edu 